\title{
A many-body approach to Hamiltonian lattice gauge field theories
}

\author{
R.F. Bishop* and Y. Xian \\ Department of Mathematics, UMIST (University of Manchester Institute of Science and Technology), \\ P.O. Box 88, Manchester M60 1QD, United Kingdom
}

The application of an extremely powerful $a b$ initio technique in microscopic many-body theory, namely the coupled-cluster method, to Hamiltonian lattice gauge field theories is outlined. Some numerical results for the $U(1)$ model in $2+1$ dimensions are presented.

Lattice gauge field theory can most readily be treated as a many-body problem when it is formulated in a Hamiltonian form. For example, for the Abelian $U(1)$ model, the group element on a link $l$ originating at a lattice site with position vector $\mathbf{n}$ in (positive) direction $k$ may be written as $U_{k}(\mathbf{n})=\exp \left[i A_{k}(\mathbf{n})\right]$, and the corresponding Hamiltonian as,

$H=\frac{1}{2} \sum_{k, \mathbf{n}} E_{k}^{2}(\mathbf{n})+\lambda \sum_{k, \mathbf{n}}\left[1-\cos B_{k}(\mathbf{n})\right]$,

where $E_{k}(\mathbf{n})$ is the electric field on the links and $B_{k}(\mathbf{n})$ is the magnetic field defined as the lattice curl, $B_{i}(\mathbf{n})=\epsilon_{i j k}\left[A_{k}\left(\mathbf{n}+\mathbf{e}_{j}\right)-A_{k}(\mathbf{n})\right]$, around the elementary plaquettes defined by the unit lattice vectors $\mathbf{e}_{i}$. Quantum mechanics is imposed in the temporal gauge via the fundamental commutation relation, $\left[A_{k}(\mathbf{n}), E_{k^{\prime}}\left(\mathbf{n}^{\prime}\right)\right]=$ $i \delta_{k k^{\prime}} \delta_{\mathbf{n n}}$, which may be realized by the representation $E_{k}(\mathbf{n}) \rightarrow-i \partial / \partial A_{k}(\mathbf{n})$. It is therefore not difficult to write $H$ wholly in terms of plaquette variables, $B_{p}\left(=B_{k}(\mathbf{n})\right)$, in the gauge-invariant sector. In $2+1$ dimensions, for example, we find

$$
\begin{gathered}
H=\sum_{p}\left[-2 \frac{\partial^{2}}{\partial B_{p}^{2}}+\lambda\left(1-\cos B_{p}\right)\right] \\
+\sum_{\left.<p, p^{\prime}\right\rangle} \frac{\partial^{2}}{\partial B_{p} \partial B_{p^{\prime}}}
\end{gathered}
$$

where the second sum over $\left\langle p, p^{\prime}\right\rangle$ indicates all nearest-neighbour pairs of plaquettes. The

\footnotetext{
*This work is supported by a research grant from SERC
} (Great Britain).
Hamiltonian of Eq. (2) thus defines an infinite many-body problem with compact variables, $-\pi<B_{p} \leq \pi$.

In the non-Abelian $S U(N)$ case the basic variables are the $S U(N)$ matrices defined on each link $l$ in terms of the $N^{2}-1$ group generators, and the conjugate "chromoelectric" fields $E_{l}^{\alpha}$ have $N^{2}-1$ components. For example, in the case of $S U(2)$, the group element on each link has the general form $U=d_{0} 1+i \mathbf{d} \cdot \sigma$, where the $\sigma_{k}$ are the usual Pauli matrices, and the real coefficients $d_{\alpha}$ satisfy $d_{0}^{2}+\sum_{k=1}^{3} d_{k}^{2}=1$, and hence lie on a sphere in four-dimensional Euclidean space. The potential term in Eq. (1) generalizes to $\lambda \sum_{p}\left(N-\operatorname{Re} \operatorname{Tr} U_{p}\right)$, where $U_{p}=U_{1} U_{2} U_{3}^{\dagger} U_{4}^{\dagger}$ for the plaquette formed from the four links $l=1,2,3,4$ in cyclic order.

Our primary aim is now to parametrize the physical (i.e., gauge-invariant) ground state and excited ("glueball") states of the above Hamiltonians in the vacuum sector, by the powerful microscopic many-body technique, the coupledcluster method (CCM), which has been widely applied in quantum chemistry, nuclear physics, and condensed matter physics [1]. The first need in our CCM analysis is to choose a suitable model state $|\Phi\rangle$, and to define the corresponding excitation operators (or correlation operators $S$ ) with respect to $|\Phi\rangle$. A convenient choice is the socalled electric $(\lambda \rightarrow 0)$ vacuum $|0\rangle$, for which $E_{l}|0\rangle=0$ for all $l$. An arbitrary excitation operator with respect to this electric vacuum is given by a sum over all possible linearly independent linked clusters of Wilson loops, $S=\sum_{\Gamma, p} \mathcal{S}_{\Gamma} A_{\Gamma}(p)$ with $\Gamma\left(\equiv\left\{\gamma_{i} ; i=1,2, \ldots, n\right\}, n=1,2, \ldots\right)$ and $p$ de- 
noting the geometry and position of the oriented loop clusters respectively, and $A_{\Gamma}=A_{\gamma_{1}} \cdots A_{\gamma_{n}}$, with $A_{\gamma}=\operatorname{Tr}[U \cdots U]_{\gamma}$ for each closed loop $\gamma$.

The CCM ansatz $[2,3]$ for the exact ground state of a given Hamiltonian $H$ is then given by the exponentiated form, $\left|\Psi_{g}\right\rangle=\exp (S)|\Phi\rangle$. The ground state energy $E_{g}$ and correlation coefficients $\left\{\mathcal{S}_{\Gamma}\right\}$ are determined by the following coupled equations respectively,

$$
\begin{aligned}
& E_{g}=\frac{\langle\Phi|\tilde{H}| \Phi\rangle}{\langle\Phi \mid \Phi\rangle} \\
& \sum_{p}\left\langle\Phi\left|A_{\Gamma}^{\dagger}(p) \tilde{H}\right| \Phi\right\rangle=0, \quad \forall \Gamma,
\end{aligned}
$$

in terms of the similarity-transformed Hamiltonian $\tilde{H}$ with the nested-commutation expansion,

$$
\tilde{H} \equiv \mathrm{e}^{-S} H \mathrm{e}^{S}=H+[H, S]+\frac{1}{2 !}[[H, S], S]+\cdots \cdot(5)
$$

For the Hamiltonian of Eq. (1), the expansion of Eq. (5) terminates at the term of second order in $S$. Finally, the inner products in Eqs. (3) and (4) incorporate the appropriate group measure.

For ease of discussion let us henceforth focus on the $U(1)$ model of Eq. (2) in 2+1 dimensions [2]. The electric vacuum state is thus a constant, and we have $|\Phi\rangle \rightarrow C$, a $c$-number. The corresponding correlation operator $S$ can be expressed, in the language of many-body theory, as a summation of $k$-plaquette correlation operators $S_{k}$, i.e., $S=$ $\sum_{k=1}^{N_{\mathbf{p}}} S_{k}$, with $N_{p} \rightarrow \infty$ the number of plaquettes in the lattice. In particular,

$$
\begin{aligned}
S_{1}= & \sum_{n=1}^{\infty} \sum_{p=1}^{N_{p}} \mathcal{S}_{p}(n) \cos n B_{p} \\
S_{2}= & \sum_{n, n^{\prime}=1}^{\infty} \sum_{p, p^{\prime}=1}^{N_{p}}\left[\mathcal{S}_{p p^{\prime}}^{(1)}\left(n, n^{\prime}\right) \cos n B_{p} \cos n^{\prime} B_{p^{\prime}}\right. \\
& \left.+\mathcal{S}_{p p^{\prime}}^{(2)}\left(n, n^{\prime}\right) \sin n B_{p} \sin n^{\prime} B_{p^{\prime}}\right]
\end{aligned}
$$

etc., where the prime on the sum in Eq. (7) excludes the term with $p=p^{\prime}$. For this case, we have $S^{\dagger}=S$, and the inner products in the coupled equations of Eqs. (3) and (4) are simply the multiple integrals over all variables $\left\{B_{p}\right\}$ within the range $-\pi<B_{p}<\pi$. We note that the many-body correlation operators $\left\{S_{k}\right\}$ have a close relation to the usual Wilson loops $A_{\Gamma}$ mentioned earlier. For example, one can write, $2 \cos B_{1} \cos B_{2}=\cos \left(B_{1}+B_{2}\right)+\cos \left(B_{1}-B_{2}\right)$, which corresponds to the following relation for the Wilson loops:

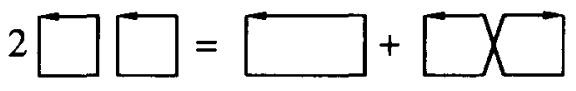

Our parametrization exemplified by Eqs. (6) and (7) is clearly complete. It is also particularly useful in view of the orthonormality of the basis.

Practically, we may implement either the standard $S U B n$ truncation scheme in which one approximates the infinite sum in $S$ as $S \rightarrow S_{\mathrm{SUB} n}=$ $\sum_{k=1}^{n} S_{k}$, and sets $S_{m}=0$ for all $m>n$; or a new local truncation scheme, called LSUB $n$, where we neglect not only correlations between $m$ plaquettes with $m>n$ but also correlations between $m<n$ plaquettes if those $m$ plaquettes occupy a region on the lattice which cannot be delimited by at most $n$ contiguous plaquettes. Further subtruncations in both schemes can be performed in terms of the number of modes $\left\{n_{i}\right\}$ kept in the sums in Eq. (6) and (7), e.g., the $\operatorname{LSUB} n(m)$ scheme where one ignores those terms in the LSUB $n$ correlation operator with $\sum_{k} n_{k}>m$. We should point out that these mode numbers $\left\{n_{i}\right\}$ bear a direct relationship with the winding numbers of the equivalent Wilson loops retained in the CCM approximation for $S$. Indeed, in this latter context, it should be clear how to generalize our approach to $S U(N)$ lattice gauge field theory. Details of the truncation schemes in our CCM calculations can be found in Refs. [2-4].

In Figure 1 and Table 1 we show the groundstate energy as a function of $\lambda$ in our SUB1 and LSUB2 $(m)$ (with $m \leq 10$ ) approximations. The SUB1 calculation exactly reproduces the onebody Mathieu problem. We also include for comparison in Figure 1 the results of $n$ th-order strong-coupling perturbation theory (denoted as PT $n$ ), with $n \leq 8$; and in Table 1 , the results from an analytic continuation of the strong-coupling perturbation series due to Hamer, Oitmaa and Zheng (denoted as HOZ) [5], and from the $t$ expansion calculation of Morningstar [6]. One sees clearly from these results that our LSUB2 $(m)$ 
Table 1

Ground-state energy per plaquette at several values of $\lambda$ for the $U(1)$ model on the square lattice.

\begin{tabular}{lccccccccc}
\hline & \multicolumn{10}{c}{$\lambda$} & \multicolumn{10}{c}{$\lambda$} \\
\cline { 2 - 10 } Method & 0.5 & 1 & 2 & 3 & 4 & 5 & 6 & 8 & 9 \\
\hline SUB1 & 0.4391 & 0.7724 & 1.2430 & 1.5828 & 1.8597 & 2.1000 & 2.3156 & 2.6966 & 2.8688 \\
LSUB2(2) & 0.4386 & 0.7652 & 1.1468 & 1.1280 & 0.3019 & -2.833 & -15.11 & & \\
LSUB2(3) & 0.4387 & 0.7681 & 1.2216 & 1.5371 & 1.7691 & 1.9282 & 2.0153 & 1.9720 & 1.8424 \\
LSUB2(4) & 0.4387 & 0.7681 & 1.2214 & 1.5428 & 1.7994 & 2.0123 & 2.1921 & 2.4585 & 2.5568 \\
LSUB2(6) & 0.4387 & 0.7681 & 1.2217 & 1.5453 & 1.8099 & 2.0404 & 2.2482 & 2.6188 & 2.7886 \\
LSUB2(8) & 0.4387 & 0.7681 & 1.2217 & 1.5454 & 1.8100 & 2.0404 & 2.2477 & 2.6142 & 2.7797 \\
LSUB2(10) & 0.4387 & 0.7681 & 1.2216 & 1.5452 & 1.8095 & 2.0393 & 2.2456 & 2.6096 & 2.7734 \\
HOZ & & & 1.215 & & 1.785 & & 2.2 & & \\
Morningstar & & 0.7675 & & & 1.796 & & & & 2.763 \\
\hline
\end{tabular}

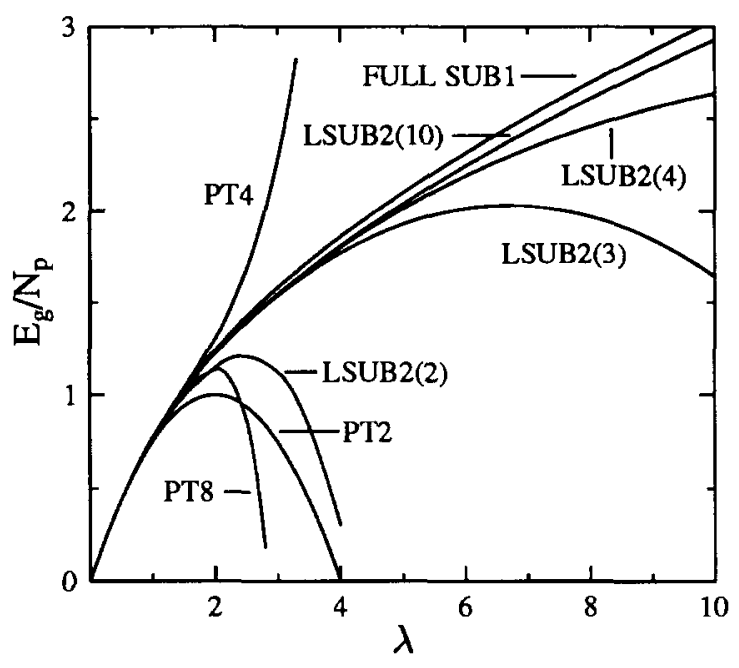

Figure 1. Ground-state energy per plaquette of the $U(1)$ model on the square lattice.

results quickly converge as $m$ increases, and the LSUB2(10) results are in good agreement with those from the careful analysis of Morningstar [6]. We should also point out that the strong-coupling perturbation series can be easily reproduced from our LSUB $n$ equations [2,3]. Thus, our LSUBn approximations comprise, in effect, a well-defined analytical continuation or resummation of such perturbation series, within the context of a natural and consistent hierarchy. They are far superior to the usual ad hoc approaches based on gen- eralized Padé approximants. Preliminary work on the excitation gap (glueball mass) [3] and on the non-Abelian $S U(2)$ model [4] has also been carried out within the vacuum sector. The generalization of our above formalism for the vacuum sector to the charged sector can also be simply done in principle by including in the excitation operator $S$ sums not only over closed paths (Wilson loops) on the lattice, but also over open paths representing tubes of electric flux between staggered fermions. We hope to report results for the charged sector soon.

\section{REFERENCES}

1. R.F. Bishop and H.G. Kümmel, Phys. Today 40-3 (1987) 52; R.F. Bishop, Theor. Chim. Acta 80 (1991) 95.

2. R.F. Bishop and Y. Xian, Acta Phys. Polonica B 24 (1993) 541.

3. R.F. Bishop, A.S. Kendall, L.Y. Wong, and Y. Xian, Phys. Rev. D 48 (1993) 887.

4. R.F. Bishop and Y. Xian, in Condensed Matter Theories, Vol. 9, eds. J.W. Clark, A. Sadiq, and K.A. Shoab (Nova, New York, 1994) - to be published.

5. C.J. Hamer, J. Oitmaa, and W.H. Zheng, Phys. Rev. D 45 (1991) 4652.

6. C.J. Morningstar, Phys. Rev. D 46 (1992) 824. 